\title{
Preirradiation Graft Polymerization of Styrene in a Poly(tetrafluoroethylene) Film Investigated by Time-Resolved Small-Angle Neutron Scattering
}

\author{
Hiroki Iwase, ${ }^{1}$ Shin-ichi Sawada,, ${ }^{2}$ Tetsuya Yamaki, ${ }^{2}$ Yasunari Maekawa, ${ }^{2}$ \\ and Satoshi Koizumi ${ }^{1}$ \\ ${ }^{1}$ Quantum Beam Science Directorate, Japan Atomic Energy Agency, Ibaraki, Tokai 319-1195, Japan \\ ${ }^{2}$ Quantum Beam Science Directorate, Japan Atomic Energy Agency, Gunma, Takasaki 370-1292, Japan \\ Correspondence should be addressed to Satoshi Koizumi, koizumi.satoshi@jaea.go.jp
}

Received 18 January 2011; Revised 3 May 2011; Accepted 1 June 2011

Academic Editor: Wen Fu Lee

Copyright (๑) 2011 Hiroki Iwase et al. This is an open access article distributed under the Creative Commons Attribution License, which permits unrestricted use, distribution, and reproduction in any medium, provided the original work is properly cited.

Preirradiation graft polymerization of styrene in a poly(tetrafluoroethylene) (PTFE) film was examined by time-resolved smallangle neutron scattering (SANS). A crosslinked PTFE film, thickness of which is about $50 \mu \mathrm{m}$, was irradiated by $\gamma$-ray and immersed in a mixed solvent of styrene monomer and toluene. SANS elucidated that graft polymerization proceeds by two reaction processes (I) and (II). In process (I) at $0<t<200 \mathrm{~min}$, graft polymerization occurs at an interface between crystalline and amorphous PTFE domains and the grafted polystyrene segregates from PTFE, forming a thin layer with a sharp interface. In process (II) at $200<t<600 \mathrm{~min}$, grafted PS layer starts to bridge between crystalline domains. At the end of process (II), $40 \%$ of total crystalline PTFE domain is covered by the grafted PS chains.

\section{Introduction}

Preirradiation polymerization, using ionizing radiation, was intensively explored in the middle of twentieth century [1]. The Preirradiation method can homogeneously produce free radicals in a solid film specimen. Using a free radical as an active site, graft polymer chains can be introduced on backbone chains composed of a film specimen. It is commercially and industrially available to functionalize a hydrocarbon polymer $[2,3]$ or particularly to modify a fluoropolymer [4$6]$ or engineering plastics $[7,8]$.

In this paper, we investigate Preirradiation polymerization of styrene onto poly(tetrafluoroethylene) (PTFE) film, motivated by an excellent picture (Figure 1 in [8]). PTFE is well known as an inert material to essentially all common chemicals, except for molten alkali metals or pure oxygens at elevated temperature. PTFE usually forms crystalline structure, which further reinforces chemical resistance. However, when a PTFE film is irradiated by ionizing radiation (step (2) in Figure 1(a)) and immersed in styrene (St) monomers, the film starts to be swollen by St monomers and graft polymerization occurs homogeneously throughout a whole film. This radical polymerization continues to be living for hours, days, or sometimes months. Consequently, a swelling ratio after polymerization reaches to about 10 as compared to an initial state before polymerization. PTFE is modified into polymer electrolyte membranes useful for electrochemical applications when the grafted PS chain is sulfonated (step (3) in Figure 1(a)).

In a course of history of radiation chemistry, it was experimentally confirmed that free radicals induced by ionizing irradiation are stabilized in a solid phase of crystal, in which a mobility of polymer chains is strongly restricted so that a recombination reaction between radicals is difficult to occur (see Figure 1(b)). Monomers, on the other hand, diffuse only into an amorphous matrix from a film surface. When a free radical in a crystalline domain migrates to an interface between crystalline and amorphous domains, it encounters a monomer. Then, graft polymerization starts to occur at the interface between crystalline and amorphous domains. 
This scenario, describing Preirradiation polymerization in a crystalline film, involves interdisciplinary scientific topics, such as (i) production, stabilization, and diffusion of free radicals in a crystalline PTFE domain, (ii) radical polymerization, (iii) phase separation between PTFE and PS chains, and (iv) deformation and breakage of a film by swelling monomer or grafted polymers. These individual processes are coupled with each other to control a radical polymerization behavior. Especially, the microstructure in a film specimen plays an important role. Thus, knowledge based on radiation chemistry, physical chemistry, and polymer chemistry is necessary to be combined to understand the Preirradiation polymerization.

To elucidate Preirradiation polymerization of PTFE, an in situ and real-time structural analysis during polymerization is necessary. For that purpose, we employed a timeresolved small-angle neutron scattering (SANS) method, which is suitable to monitor chemical reaction-induced selfassembled structure [9-17]. Cold neutron utilized for SANS is largely advantageous for our in situ observation. An intrinsic energy of cold neutron is of order of $0.1 \mathrm{mev}$, which is comparable to the energy of thermal vibrations in a polymer chain. Therefore, irradiation of cold neutron does not damage a polymer film by causing a side reaction. Neutron is highly permeable into a quartz vessel in which radical polymerization occurs, because it is electrically neutral. Therefore, we are able to see in a reacting solution as it is living.

Our method to prepare a polymer electrolyte from PTFE involves three steps as indicated in Figure 1(a): step (1) of crosslink reaction by electron irradiation at high temperature, step (2) of graft polymerization by Preirradiation of $\gamma$-ray, and step (3) of sulfonation of grafted PS polymer chains. In this paper, we investigated steps (1) and (2) by using SANS. As for step (1), we discuss microcrystal formation induced by crosslink of PTFE chains. In step (2), we quantitatively analyze time-resolved SANS monitoring Preirradiation graft polymerization. We found that Preirradiation process of step (2) proceeds by two steps: process I of thin layer formation by grafted PS chains surrounding a PTFE crystalline domain and process II of network formation among PTFE crystalline domains.

\section{Experimental}

2.1. Preirradiation Polymerization. A crosslinked PTFE film, as referred to c-PTFE, was provided by Hitachi Cable co. ltd, which corresponds to step (1) by electron irradiation, in Figure 1(a). PTFE in melt was crosslinked with elevating temperature close to $T_{m}$. During cooling after crosslink, PTFE chains start to form a crystalline domain, which is tightly restricted by crosslink. As step (2), the c-PTFE film, which was sealed in a vacuum quartz vessel (Figure 2), was irradiated at room temperature. $\gamma$-ray irradiation of $15 \mathrm{kGy}$ dose was performed at the Japan Atomic Energy Agency (JAEA), Takasaki, Japan. During flowing an Ar gas, St monomer was poured into a test tube and graft polymerization was initiated in a oven controlled at $60^{\circ} \mathrm{C}$.

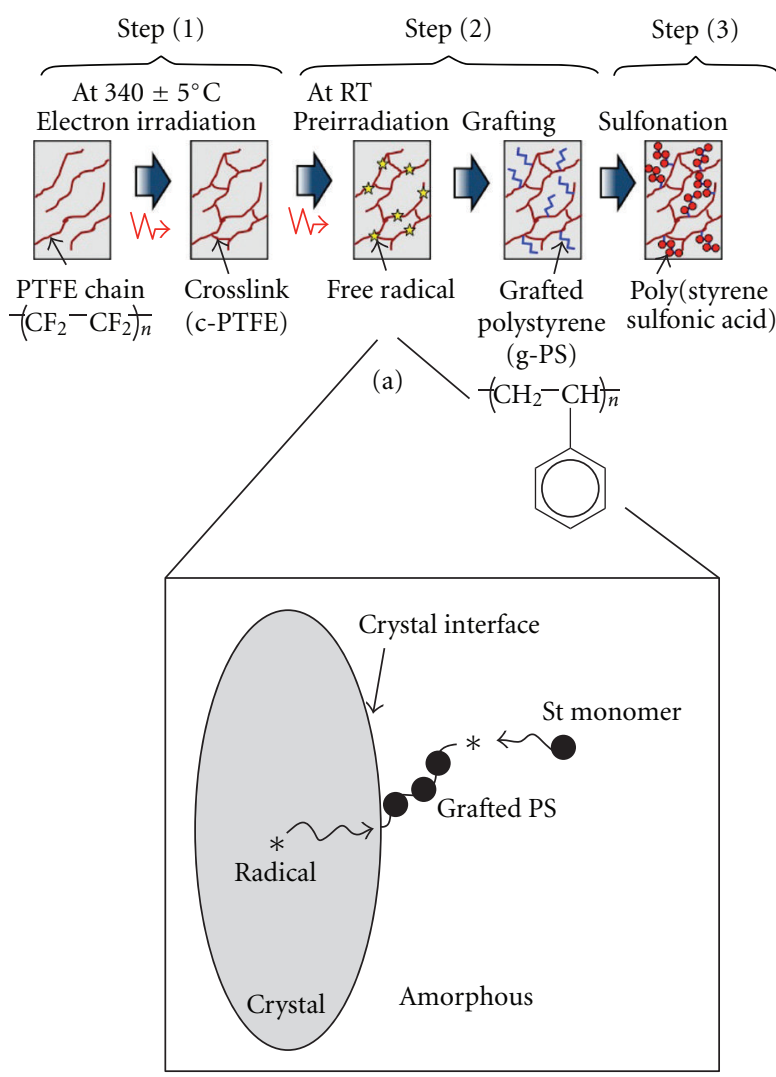

(b)

FIGURE 1: (a) A preparation procedure of polymer electrolyte membranes based on poly(tetrafluoroethylene), which is composed of three steps of (1) crosslinking, (2) grafting by Preirradiation and (3) sulfonation. (b) A schematic view of Preirradiation graft polymerization with a crystalline domain and its interface. Long living radical is accumulated in a crystalline domain, whereas monomers access from an amorphous domain.

Note that polymerization temperature of $60^{\circ} \mathrm{C}$ is between melting $\left(T_{m}\right)$ and glass transition $\left(T_{g}\right)$ temperatures of PTFE $\left(T_{m}=340\right.$ and $T_{g}=-73^{\circ} \mathrm{C}$, resp. $)$.

Graft polymerization was also monitored by weighting a film during polymerization to determine a graft polymerization ratio as a function of polymerization time $(t)$. A graft polymerization ratio is defined by $W-W_{0} / W_{0}$, where $W_{0}$ is a weight of film at $t=0$. To control the ratio, St monomer was mixed with toluene. We examined three mixed monomers of St/toluene (100/0), (66/34), and (50/50) (wt $\% / w t \%)$. SANS experiment was performed only on the c-PTFE with a mixed monomer St/toluene of (50/50) (wt\%/wt\%).

To perform time-resolved SANS observation on graft polymerization of the c-PTFE film, we prepared a special sample container, as shown in Figure 2. A c-PTFE film specimen is set in a quartz vessel, which controls a film temperature with a precision of $\pm 0.1^{\circ} \mathrm{C}$. At a top of the container, a three-way cock, made by pyrex glass, is attached in order to add St monomer and to control a vacuum condition. After $\gamma$-ray irradiation, the c-PTFE film, sealed in the sample container (Figure 2), was quenched below $-70^{\circ} \mathrm{C}$ to avoid 


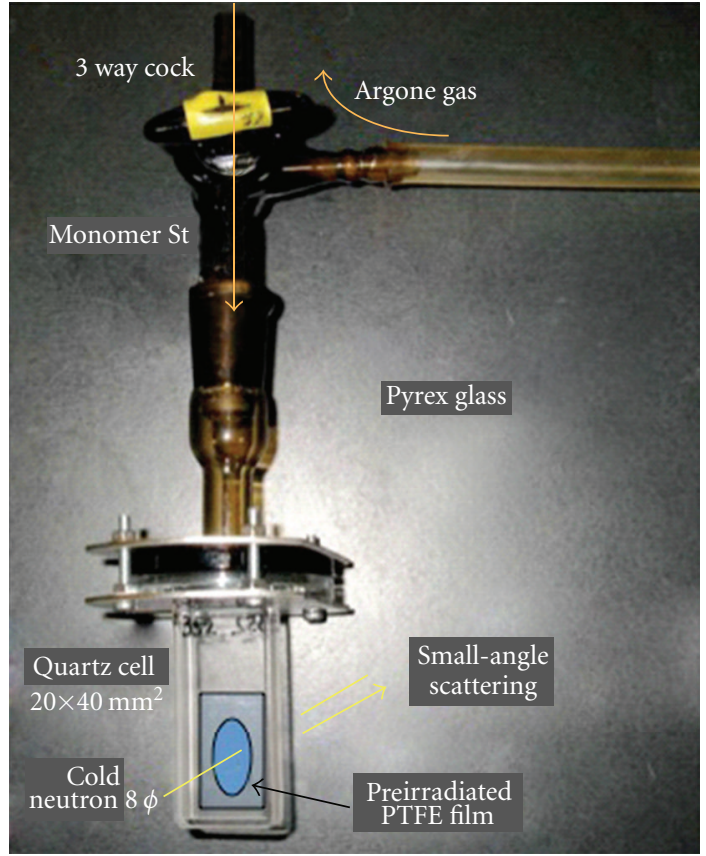

FIgURE 2: A picture of sample cell specially designed for in situ and real-time small-angle neutron scattering in order to observe Preirradiation graft polymerization on PTFE films.

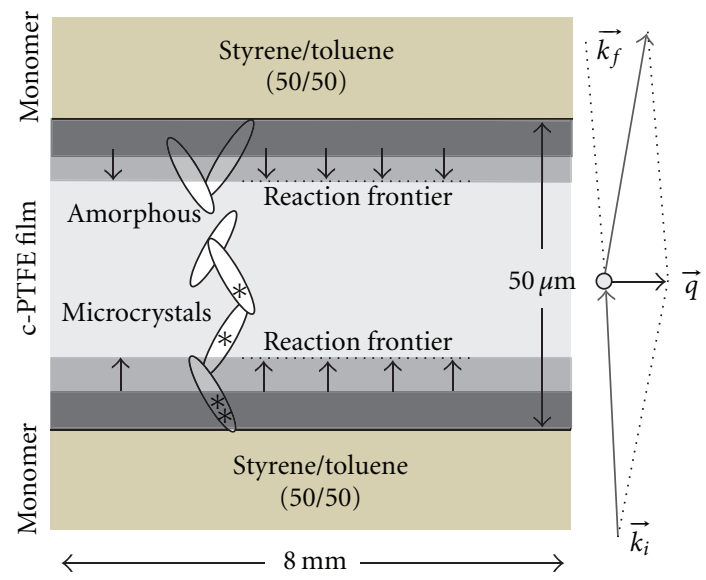

Figure 3: A schematic view showing a PTFE film during Preirradiation graft polymerization.

recombination of radicals and by spending several hours; it was transported to a research reactor JRR3 at Tokai for performing time-resolved SANS experiments.

2.2. Small-Angle Neutron Scattering. SANS measurements were performed using focusing and polarized neutron ultrasmall-angle scattering spectrometer (SANS-J-II) at research reactor JRR3, at JAEA, Toki, Japan [18]. Monochromatic cold neutron (wavelength $\lambda=0.65 \mathrm{~nm}$ and $\Delta \lambda / \lambda=15 \%$ ) was provided by a velocity selector. A measurement time for each snap shot of time-resolved SANS is $10 \mathrm{~min}$. Smallangle scattering was detected by a two-dimensional ${ }^{3} \mathrm{He}$

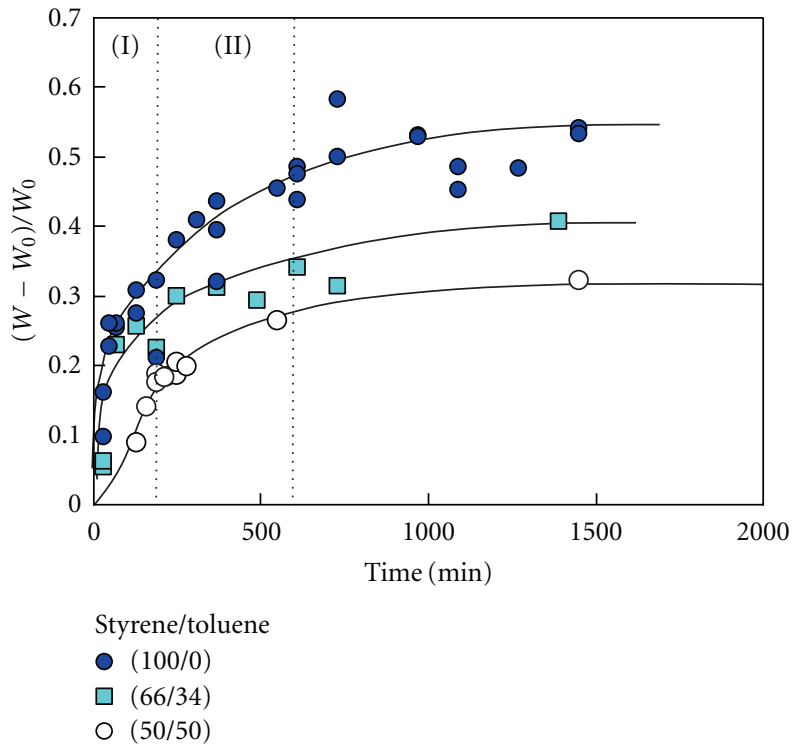

FIgure 4: Graft polymerization ratio $\left(W-W_{0} / W_{0}\right)$ as a function of polymerization time $(t)$, determined for mixed solvent of styrene/toluene (100/0), (66/34), and (50/50) (wt/wt), respectively (solid lines are guides for eye).

position-sensitive detector having a sensitive area of $65 \times$ $65 \mathrm{~cm}^{2}$ ( $128 \times 128$ pixels). Using pinhole collimations, SANSJ-II covers $q$-region from 0.03 to $1.2 \mathrm{~nm}^{-1}$ by changing a sample-to-detector distances $(10 \mathrm{~m}$ and $2.5 \mathrm{~m})$, where $q$ is the magnitude of a scattering vector as defined as $q=$ $(4 \pi / \lambda) \sin \theta$ with scattering angle $2 \theta$. The obtained twodimensional small-angle scattering was corrected as for a detector sensitivity and instrument background scattering. A secondary standard specimen of irradiated aluminum was used in order to obtain absolute scattering intensity.

As schematically shown in Figure 3, a scattering geometry is "through view," where incident neutron passes a film along its normal vector. Vectors of incident and scattered neutrons $\left(\overrightarrow{k_{i}}, \overrightarrow{k_{f}}\right)$ are almost parallel to the normal vector. Therefore, we observe microstructures in a film crossing along $q$-vector $(\vec{q})$, which is parallel to a film surface. It should be denoted that SANS observes microstructures in a reacting film, by averaging over a volume exposed for an incident neutron beam. A diameter of incident neutron is $8 \mathrm{~mm} \phi$, whereas a film thickness is $50 \mu \mathrm{m}$.

\section{Experimental Results and Discussions}

3.1. Graft Polymerization on Crosslinked Film. Figure 4 shows a graft polymerization ratio $\left[=\left(W-W_{0}\right) / W_{0}\right]$ of c-PTFE determined for three mixed monomers of St/toluene (100/0, $66 / 34$ and 50/50 wt/wt, resp.). A reaction rate for $100 / 0$ and $66 / 34$, which corresponds to an initial slope of $\left(W-W_{0}\right) / W_{0}$, is not influenced by mixing St monomer; a reaction rate for (50/50) decreases slightly. A graft polymerization ratio, on the other hand, is strongly influenced by mixing between $\mathrm{St}$ monomer and toluene. With increasing a toluene content, a graft polymerization ratio continuously decreases; it is about 


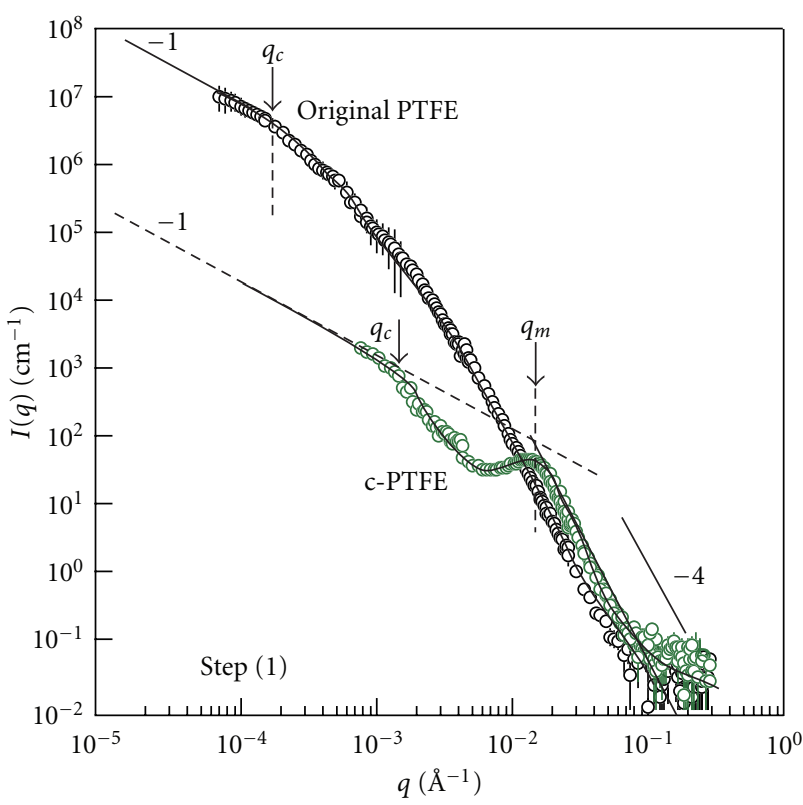

FIGURE 5: SANS obtained for crosslinked (c-PTFE) and original PTFE without crosslink (PTFE) films (solid lines are guides for eye). A crossover $q$ from $q^{-1}$ to a steeper power law is indicated by an arrow with $q_{c}$.

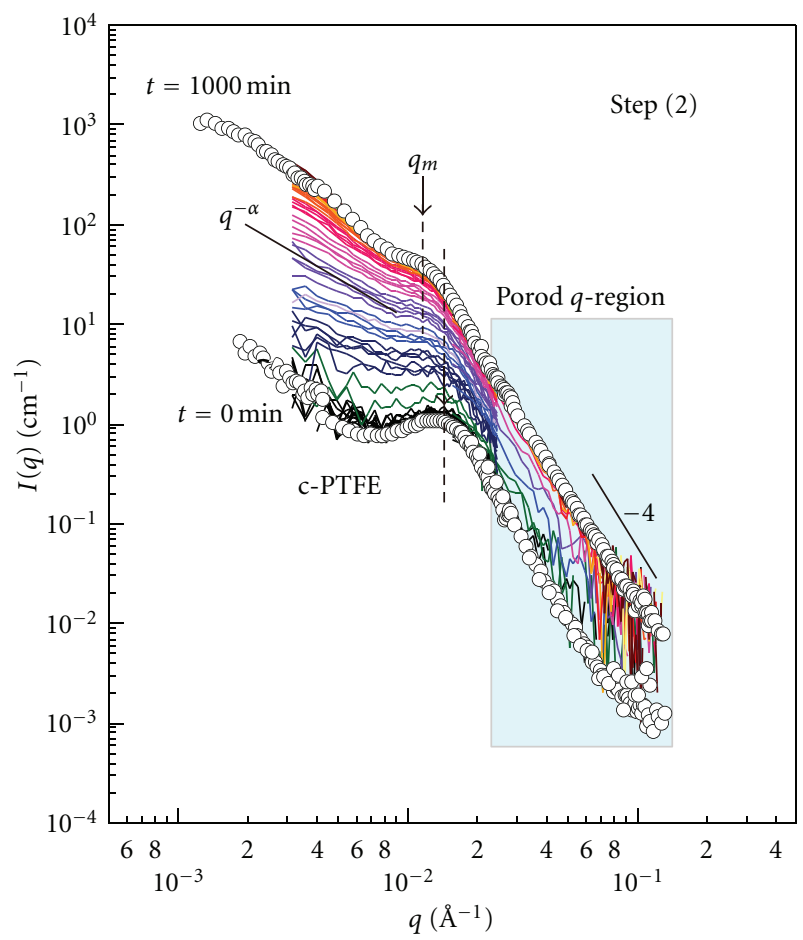

FIgURE 6: Time-resolved SANS obtained for Preirradiation graft polymerization in crosslinked (c-PTFE) (step (2)). Scattering curves indicated by open circles exhibit that for c-PTFE before polymerization and at $1000 \mathrm{~min}$ after starting polymerization. Solid lines indicate scattering curves obtained during polymerization with a measurement step of $10 \mathrm{~min}$. A Porod $q$-region showing $q^{-4}$ is indicated by a shaded region. A power law $q$-behavior $(\alpha)$ was estimated from lower $q$ according to $q^{-\alpha}$.

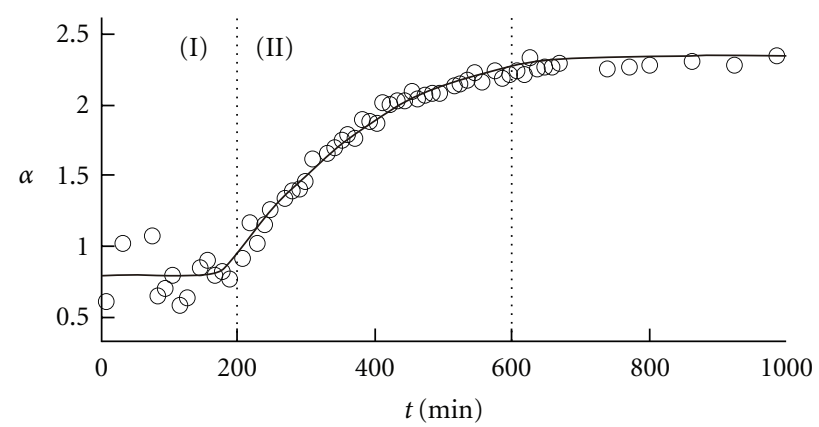

(a)

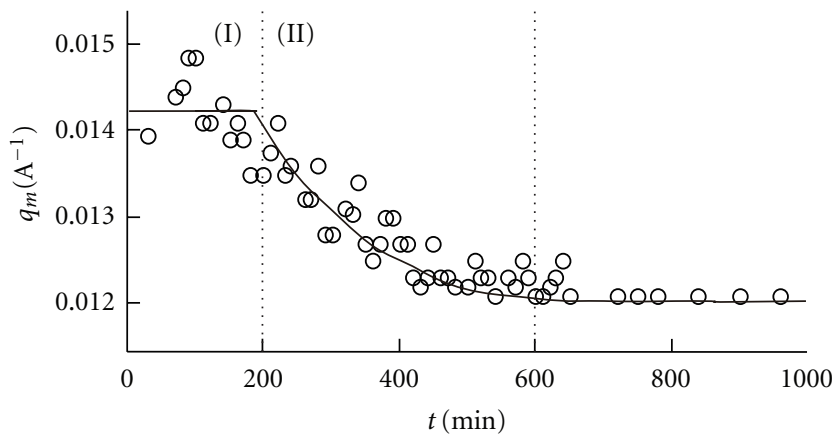

(b)

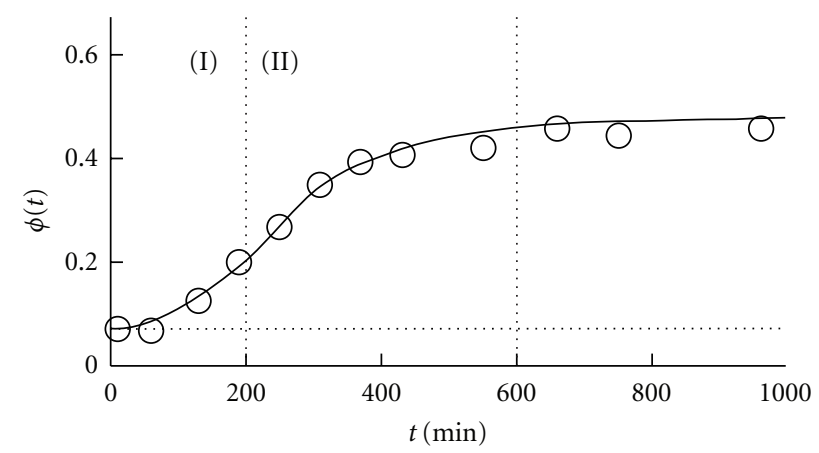

(c)

Figure 7: A power law exponent $(\alpha), q$-position of scattering maxima $\left(q_{m}\right)$, and a volume ratio of microcrystals covered by PS $\phi(t)$, determined as a function of polymerization time $(t)$.

0.5 and 0.3 for St/toluene (100/0) and (50/50), respectively. It should be denoted that when a graft polymerization ratio reaches to 0.1 , an ion conductivity across a film reaches to $0.06 \mathrm{~S} / \mathrm{cm}$, which is comparable to a Nafion [19].

\subsection{Small-Angle Neutron Scattering}

Step 1 (Microcrystal formation). The crystalline structures in the films of original PTFE without crosslink and crosslinked PTFE (c-PTFE) were examined by SANS. In Figure 5, a film specimen of the original PTFE shows SANS asymptotically changing according to $q^{-1}$ in low $q$ and $q^{-4}$ in high $q$. The asymptotic $q$-behavior of $q^{-1}$ is attributed to a needle shape of PTFE crystalline domains, whereas that of $q^{-4}$ is attributed to a sharp interface between amorphous and crystalline domains (Porod law). As shown in (see, [20, 


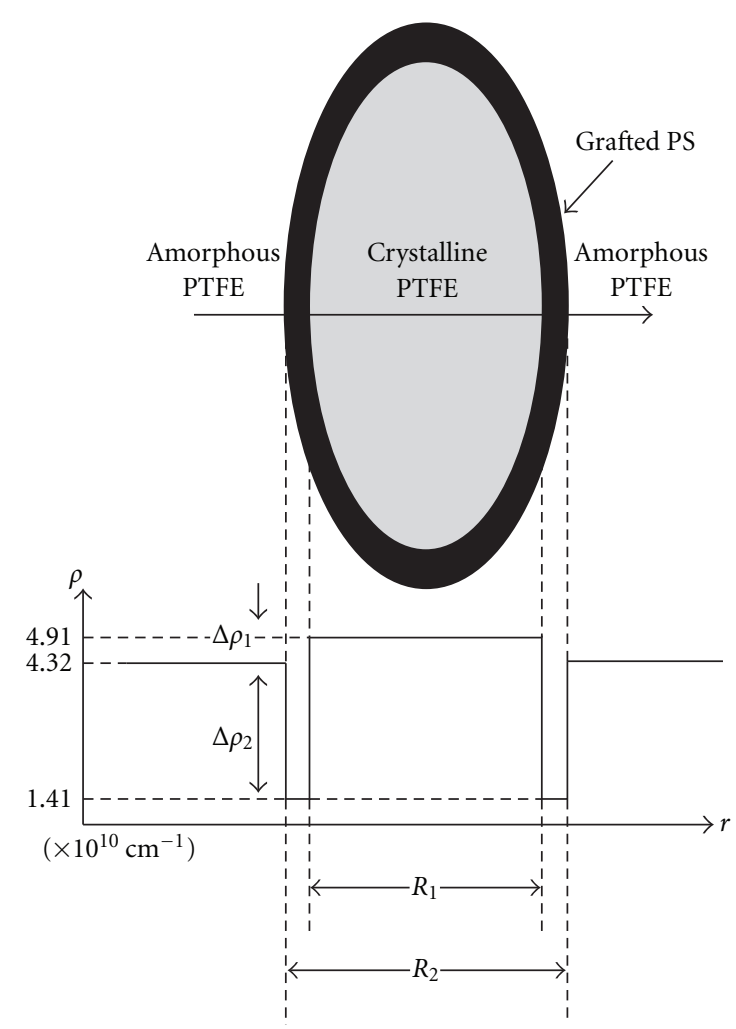

FIGURE 8: A schematic view showing an ellipsoidal PTFE microcrystal covered by PS (gray and block parts correspond to crystalline and grafted PS regions, cross-sections for which are $R_{1}$ and $R_{2}$, resp.) Coherent scattering length densities $(\rho)$ for amorphous and crystalline PTFE and grafted PS regions and their gaps $\left(\Delta \rho_{1}\right.$ and $\left.\Delta \rho_{2}\right)$ are also indicated.

Figure IV-72]), a needle-shape crystalline domain, which appears in a course of crystallization, exhibits birefringent. An asymptotic decay of $q^{-1}$ in low $q$ indicates a rod-like crystalline structure. A crossover $q\left(q_{c}=2 \times 10^{-4} \mathrm{~A}^{-1}\right)$ for power laws from -4 to -1 indicates a diameter $(R)$ of needleshape crystalline domains. We obtained $R=3 \mu \mathrm{m}\left(=2 \pi / q_{c}\right)$ for the original PTFE.

SANS obtained for the c-PTFE film exhibits a scattering maximum at $q_{m},\left(=0.014 \mathrm{~A}^{-1}\right)$, which corresponds to an interdomain distance $L=450 \mathrm{~A}\left(=2 \pi / q_{m}\right)$. In high $q$, SANS decreases monotonously according to $q^{-4}$. In low $q$, an asymptotic decay changes into $q^{-1}$. As compared to the original PTFE, $q_{c}$ for c-PTFE shifts toward high $q$, implying that $R$ becomes smaller. Crosslink of PTFE chains induced in step (1) might pin crystallization and make crystalline domains smaller (microcrystal formation). Although the crystalline domain becomes smaller for the c-PTFE, a degree of crystallinity does not change as $40 \%$, which is comparable to the original PTFE without crosslinks [21]. Decrease in crystalline domain size $(R)$ with keeping a same crystallinity causes increase of total interface area; according to SANS analyses in a Porod $q$-region, the interface area for the c-PTFE film increases as a factor of 3.4, as compared to the original PTFE film.
Step 2 (In situ and time-resolved observation). Figure 3 schematically indicates a film specimen during graft polymerization and a scattering geometry. A sheet of irradiated cPTFE film, the thickness of which is $50 \mu \mathrm{m}$, was immersed in the mixture of styrene and toluene $(50 / 50 \mathrm{wt} / \mathrm{wt})$. St monomers swells a film from its surfaces. Graft polymerization occurs from near the film surface, and a reaction frontier line (indicated by different colors in Figure 3) moves deeper into the film. During graft polymerization, SANS observes microstructures averaged over a sample volume, which is exposed by an incident neutron beam.

Graft polymerization of step (2) was monitored by timeresolved SANS. Time-dependent SANS $q$-profiles are shown in Figure 6. After an incubation time of several hours, SANS obtained for reacting c-PTFE starts to increase in its intensity, keeping its $q$-behavior identical to that before polymerization. However, if we carefully observe SANS after about $200 \mathrm{~min}$, we found that the polymerization time increases as follows. First, $q_{m}$ slightly shifts toward lower $q$, which corresponds to change in $L\left(=2 \pi / q_{m}\right)$. We evaluated $L=440$ and $520 \mathrm{~A}$ at 200 and $600 \mathrm{~min}$, respectively. Secondly, the asymptotic $q$-behavior in low $q$, which was estimated by a power law $\alpha$ according to $q^{-\alpha}$, becomes slightly steeper. Figure 7 shows $q$-positions of $q_{m}$ and power law $\alpha$ obtained in low $q$ as a function of polymerization time. From $0<t<$ $200 \mathrm{~min}$ (we denote region (I)), $q_{m}$ remains constant, whereas for $t>200 \mathrm{~min}$ (we denote region (II)), $q_{m}$ starts to shift to low $q$. A similar tendency was found for $\alpha$; in region (I), $\alpha$ is constant $(\alpha=1)$, whereas in region (II), $\alpha$ starts to increase to $\alpha=2.5$ from 1 .

In high $q$, SANS $q$-profiles obtained at different polymerization times show an asymptotic decay according to $q^{-4}$, which is a Porod law, attributed to formation of sharp interface. Before starting graft polymerization, the Porod law originates from a sharp interface between crystalline and amorphous PTFE domains in a c-PTFE film. A difference in density between crystalline and amorphous PTFE domains causes a scattering contrast $\left(\Delta \rho_{1}\right.$ in Figure 8$)$. After staring graft polymerization, the PS chains, grafted at the interface of crystalline domain, segregate from both of amorphous and crystalline PTFE domains and result in a sharp thin PS layer (a black region in Figure 8). The sharp interface of thin PS layer again causes small-angle scattering according to $q^{-4}$.

Model Analysis on SANS. In order to quantitatively discuss time-resolved SANS, we employ a scattering model considering a core/shell structure for a grafted crystalline domain; a core and shell correspond to crystalline PTFE domain and grafted PS thin layer. In a PTFE film specimen, ellipsoidal crystalline domains are dispersed in an amorphous matrix. The cross-section of crystalline domain is denoted as $R_{1}$ in Figure 8. Because of difference in density between crystalline and amorphous PTFE domains $\left(\rho_{d}=2.0\right.$ and $2.3 \mathrm{~g} / \mathrm{cm}^{3}$, resp.), scattering length densities for crystalline core and amorphous matrix are given as $\rho=4.91 \times 10^{10} \mathrm{~cm}^{-1}$ and $\rho=$ $4.32 \times 10^{10} \mathrm{~cm}^{-1}$, respectively. Therefore, a scattering contrasts are given as $\Delta \rho_{1}=0.59 \times 10^{10} \mathrm{~cm}^{-1}$.

After starting graft polymerization, a crystalline domain starts to be covered with a thin layer of grafted PS (a black 
region in Figure 8). The cross-section of crystalline domain covered with PS is given by $R_{2}$. The thickness of thin PS layer $\left(=\left(R_{2}-R_{1}\right) / 2\right)$ might be approximated to be negligibly thin and therefore $R_{1} \cong R_{2}$. This is because $q_{m}$, as shown in Figure 7(b), does not largely change before and after graft polymerization. According to SANS observations in a Porod $q$-region at high $q$ (shaded $q$-region in Figure 6), PS graft chains segregate from PTFE domains so that the interface between core and shell is flat and sharp.

Small-angle neutron scattering intensity $I(q)$ is composed of a form factor for needle-shape microcrystal covered with a PS thin layer $F(q)^{2}$ and its structure factor $S(q)$, which describes a spatial distribution of the microcrystals. $I(q)$ is given by

$$
I(q)=K n_{c} V_{c}^{2} F(q)^{2} S(q),
$$

where $n_{c}$ and $V_{c}$ are a number density and volume of a PTFE microcrystal. $K$ in (1) denotes an instrument constant. The scattering maximum at $q_{m}$ observed in Figures 5 and 6 is attributed to an interparticle interference resulting in $S(q)$. A Porod $q$-behavior of $q^{-4}$ found at high $q$ is attributed to $F(q)^{2}$ in $(1)$.

In our analysis, we postulate that $n_{c}$ and $V_{c}$ do not change during graft polymerization. For a microcrystal covered with a PS thin layer (see Figure 8), we obtain $F(q)^{2}$, as follows:

$$
F(q)^{2}=\left[\Delta \rho_{1} f\left(q, R_{1}\right)-\Delta \rho_{2} f\left(q, R_{2}\right)\right]^{2},
$$

where $f(q, R)$ describes a form factor of ellipsoid domains with a minor axis $R$. If we employ $\Delta \rho_{2}=0$ in (2), we obtain $f(q)$ for a crystalline domain without a PS thin layer.

At the beginning of graft polymerization with $R_{1} \cong R_{2}$, (1) is rewritten as

$$
F(q)^{2} \cong\left(\Delta \rho_{1}-\Delta \rho_{2}\right)^{2} f\left(q, R_{1}\right)^{2} .
$$

In a Porod $q$-region, we approximate $f(q, R)$ as follows:

$$
F(q)^{2}=\left(\Delta \rho_{1}-\Delta \rho_{2}\right)^{2} S_{V} q^{-4},
$$

where $S_{V}$ is a total surface area of all PTFE crystalline domains in a scattering volume.

According to an illustration in Figure 3, describing the graft polymerization of PS in a film specimen, a monomer diffuses into the film from its surface and the reaction starts to occur from near the film surface. In a course of graft polymerization, first PTFE microcrystals, existing near the film surface, are covered by PS chains. The PTFE microcrystals, existing deeply in the film, are not covered. To describe this situation, we introduce a parameter $\phi(t)$, which gives a fraction of covered PTFE microcrystals during graft polymerization. Then, small-angle scattering in a Porod $q$-region is given by

$$
\begin{aligned}
I(q, t) \propto & {\left[\left(\Delta \rho_{1}-\Delta \rho_{2}\right)^{2}-\Delta \rho_{1}^{2}\right] } \\
& \times S_{V} q^{-4}\left\{\phi(t)+\left[\frac{\left(\Delta \rho_{1}-\Delta \rho_{2}\right)^{2}}{\Delta \rho_{1}^{2}}-1\right]^{-1}\right\} .
\end{aligned}
$$

According to (5), we find that time evolution of SANS intensity in a Porod $q$-region is proportional to $\phi(t)$.

By using (5), we examined a Porod $q$-region and evaluated $\phi(t)$ (Figure 7). From the beginning of graft polymerization, $\phi(t)$ starts to increase and finally after $t=600 \mathrm{~min}$ it reaches to $40 \%$. On the other hand, $\alpha$ and $q_{m}$, which start to increase from $t=200 \mathrm{~min}$, become time independent at $t=$ $600 \mathrm{~min}$.

On a basis of time-resolved SANS observation and its model analysis, we illustrate a mechanism of graft polymerization in the c-PTFE film. We consider two processes (I) before $t=200 \mathrm{~min}$ and (II) after $t=200 \mathrm{~min}$, based on time evolution of $q_{m}$ and $\alpha$. In process (I), the grafted PS forms a thin layer at the interface of PTFE crystalline domain. A thin layer grows along on the interface of crystalline PTFE domain. The increase of SANS intensity in process (I) is attributed to the increase of $\phi(t)$ ( or $S_{V}, \phi(t)$, total surface area covered by PS graft chains). The area of process (I) starts to appear first near the film surface and migrates deeper into the film. In process (I), the interdomain distance $L$ (= $\left.2 \pi / q_{m}\right)$ between crystalline PTFE domains does not change (as observed by $q_{m}$ in Figure 7 ). As shown in Figure 4 , a graft polymerization ratio reaches to about 0.2 in a process (I), which results in sufficiently large ion conductivity $(\sim 0.06 \mathrm{~S} / \mathrm{cm})$ for a film thickness direction.

In process (II), it is supposed that the grafted PS chains (or thin layer) form a bridge between crystalline PTFE domains. The bridges, growing toward a direction along a normal vector of the interface, percolate among crystalline PTFE domains. A power law $\alpha$ determined by SANS might be interpreted as mass fractal dimension $\left(d_{m}\right)$ of PS percolation, where $d_{m}$ is identical with $\alpha\left(\alpha=d_{m}\right)$ [22]. In process (II), the interdomain distance $L\left(=2 \pi / q_{m}\right)$ starts to slightly increase.

To largely swell a PTFE film during Preirradiation graft polymerization, deformation and breakage of a PTFE film are necessary. When PTFE is irradiated by ionizing radiation below a melting temperature $\left(T<T_{m}=340^{\circ} \mathrm{C}\right)$, a PTFE chain suffers from scission. According to electron-spin resonance (ESR), $\beta$-scission dominantly occurs and an end-chain radical is created [23]. It is reported that, at $t=200 \mathrm{sec}$ after $\gamma$-ray irradiation, a molar fraction of end-chain radical is maximized and, at $8000 \mathrm{sec}$, it is stabilized. The end-chain radical is transferred to another chain to cause successive $\beta$ scission. Thus, PTFE chains are segmented. We suppose that process (I) found by SANS corresponds to a time domain of $\beta$-scission.

Due to strong segregation between grafted PS and PTFE, a thin layer of grafted PS has a sharp interface, which results in small-angle scattering of $q^{-4}$. The mixed solvent of toluene, which is a good solvent for PS, swells the PS thin layer so that St monomer diffusion might be more smoothly in the PS thin layer. The mobility of PS chain is also enhanced so that recombination termination becomes more frequent, which decreases a graft polymerization ratio and its rate, as shown in Figure 3. It is reported that if we mix a poor solvent (such as alcohol or water) with St monomer, a graft polymerization ratio is increased as compared to a good solvent (e.g., toluene used in this study) [24]. A poor solvent 
Decreases a swelling degree of PTFE film, so that the mobility of grafted polymer chains is also decreased.

\section{Conclusion}

We investigated Preirradiation graft polymerization of styrene in a crosslinked poly(tetrafluoroethylene) (c-PTFE) film by time-resolved SANS. It was elucidated that graft polymerization of PS proceeds by two steps: first, as process (I), graft polymerization occurs at an interface of PTFT microcrystal and a thin layer of pure PS chains is formed to surround the PTFE microcrystal. Second, as process (II), grafted PS chains or thin layer starts to bridge between crystalline domains. At the end of process (II), $40 \%$ of total microcrystal is covered by the PS thin layer.

\section{References}

[1] A. Chapiro, Radiation Chemistry of Polymeric Systems, Interscience Publishers, London, UK; John Wiley \& Sons, New York, NY, USA, 1962.

[2] I. Smit and A. Bezjak, "Structural changes in the grafted copolymer polyethylene-styrene," Polymer, vol. 22, no. 5, pp. 590-596, 1981.

[3] T. Seguchi and N. Tamura, "Mechanism of decay of alkyl radicals in irradiated polyethylene on exposure to air as studied by electron spin resonance," The Journal of Physical Chemistry, vol. 77, no. 1, pp. 40-44, 1973.

[4] T. R. Dargaville, G. A. George, D. J. T. Hill, and A. K. Whittaker, "High energy radiation grafting of fluoropolymers," Progress in Polymer Science, vol. 28, no. 9, pp. 1355-1376, 2003.

[5] S. Takahashi, H. Okonogi, T. Hagiwara, and Y. Maekawa, "Preparation of polymer electrolyte membranes consisting of alkyl sulfonic acid for a fuel cell using radiation grafting and subsequent substitution/elimination reactions," Journal of Membrane Science, vol. 324, no. 1-2, pp. 173-180, 2008.

[6] G. Gebel, F. Ottomani, J. J. Allegraud, N. Betz, and A. L. Moël, "Structural study of polystyrene grafted in irradiated polyvinylidene fluoride thin films," Nuclear Instruments and Methods in Physics Research, vol. 105, no. 1-4, pp. 145-149, 1995.

[7] S. Hasegawa, S. Takahashi, H. Iwase et al., "Radiation-induced graft polymerization of functional monomer into poly(ether ether ketone) film and structure-property analysis of the grafted membrane," Polymer, vol. 52, no. 1, pp. 98-106, 2011.

[8] A. Chapiro, "Préparation des copolymères greffés du polytetrafluoroéthylène (Teflon) par voie radiochimique," Journal of Polymer Science, vol. 34, no. 127, pp. 481-501, 1959.

[9] K. Yamauchi, H. Hasegawa, T. Hashimoto, H. Tanaka, R. Motokawa, and S. Koizumi, "Direct observation of polymerization-reaction-induced molecular self-assembling process: In-situ and real-time SANS measurements during living anionic polymerization of polyisoprene-block-polystyrene," Macromolecules, vol. 39, no. 13, pp. 4531-4539, 2006.

[10] Y. Zhao, H. Tanaka, N. Miyamoto, S. Koizumi, and T. Hashimoto, "Combined SANS, SEC, NMR, and UV-Vis studies of simultaneous living anionic copolymerization process: simultaneous elucidation of propagating living chains at three different length scales," Macromolecules, vol. 42, no. 5, pp. 17391748, 2009.

[11] Y. Zhao, N. Miyamoto, S. Koizumi, and T. Hashimoto, "Combined SANS, SEC, NMR and UV-vis studies of simultaneous living anionic copolymerization process in a concentrated solution: elucidation of building-up processes of molecules and their self-assemblies," Macromolecules, vol. 43, no. 6, pp. 2948-2959, 2010.

[12] T. Terashima, R. Motokawa, S. Koizumi et al., "In situ and time-resolved small-angle neutron scattering observation of star polymer formation via arm-linking reaction in ruthenium-catalyzed living radical polymerization," Macromolecules, vol. 43, no. 19, pp. 8218-8232, 2010.

[13] R. Motokawa, S. Koizumi, T. Hashimoto, M. Annaka, and T. Nakahira, "Soap-free emulsion polymerization of poly(ethylene glycol)block-poly( $\mathrm{N}$ - isopropylacrylamide): elucidation of specific reaction fields for quasi-living polymerization by time-resolved SANS," Macromolecules, vol. 43, no. 2, pp. 752764, 2010.

[14] H. Tanaka, S. Koizumi, T. Hashimoto et al., "Combined in situ and time-resolved SANS and SAXS studies of chemical reactions at specific sites and self-assembling processes of reaction products: reduction of palladium ions in self-assembled polyamidoamine dendrimers as a template," Macromolecules, vol. 40, no. 12, pp. 4327-4337, 2007.

[15] S. Koizumi, Y. Zaho, Y. Tomita et al., "Bacterium organizes hierarchical amorphous structure in microbial cellulose," European Physical Journal E, vol. 26, no. 1-2, pp. 137-142, 2008.

[16] S. Koizumi, Y. Tomita, T. Kondo, and T. Hashimoto, "What factors determine hierarchical structure of microbial cellulose-interplay among physics, chemistry and biology," Macromolecular Symposia, vol. 279, no. 1, pp. 110-118, 2009.

[17] H. Tanaka, S. Koizumi, T. Hashimoto, K. Kurosaki, and S. Kobayashi, "Self-assembly of synthetic cellulose during invitro enzymatic polymerization process as studied by a combined small-angle scattering method," Macromolecules, vol. 40, no. 17, pp. 6304-6315, 2007.

[18] S. Koizumi, H. Iwase, J. Suzuki et al., "Focusing and polarized neutron small-angle scattering spectrometer (SANS-J-II). The challenge of observation over length scales from an ångström to a micrometre," Journal of Applied Crystallography, vol. 40, supplement 1, pp. s474-s479, 2007.

[19] M. Asano, J. Chen, and Y. Maekawa, "Novel UV-induced photografting process for preparing poly(tetrafluoroethylene)based proton-conducting membranes," Journal of Polymer Science, Part A, vol. 45, no. 13, pp. 2624-2637, 2007.

[20] N. K. J. Symons, "Growth of single crystals of polytetrafluoroethylene from the melt," Journal of Polymer Science Part A, vol. 1, no. 9, pp. 2843-2856, 1963.

[21] T. Yamaki, K. Kobayashi, M. Asano, H. Kubota, and M. Yoshida, "Preparation of proton exchange membranes based on crosslinked polytetrafluoroethylene for fuel cell applications," Polymer, vol. 45, no. 19, pp. 6569-6573, 2004.

[22] D. Avnir, Ed., The Fractal Approach to Heterogeneous Chemistry, John Wiley \& Sons, 1989.

[23] S. Shimada and K. Yamamoto, "Mechanism of decay reaction of free radicals trapped in poly(tetrafluoroethylene) related with the heterogeneity in structure," Macromolecules, vol. 36, no. 15, pp. 5661-5665, 2003.

[24] T. Rager, "Pre-irradiation grafting of styrene/divinylbenzene onto poly(tetrafluoroethylene-co-hexafluoropropylene) from non-solvents," Helvetica Chimica Acta, vol. 86, no. 6, pp. 19661981, 2003. 

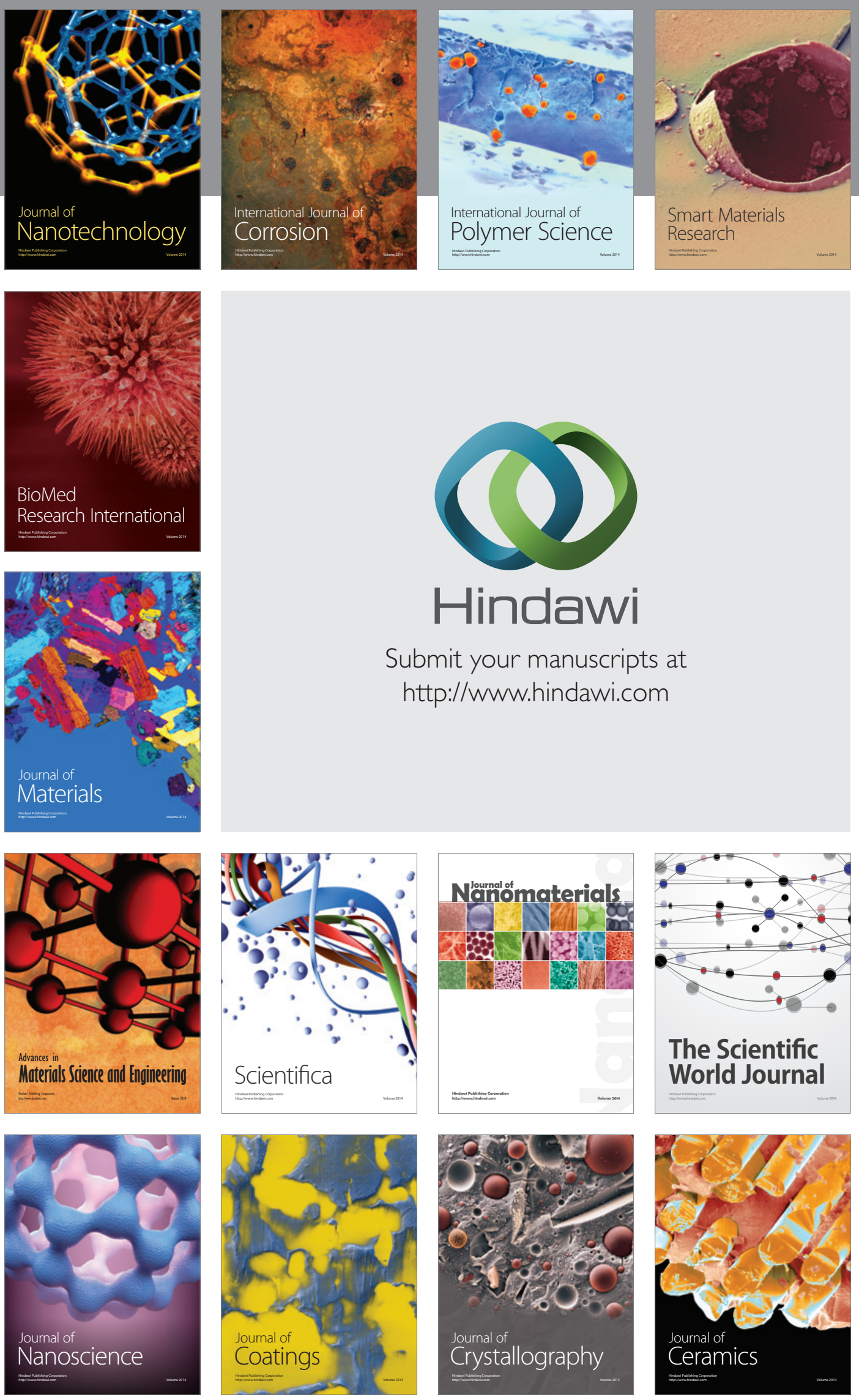

The Scientific World Journal

Submit your manuscripts at

http://www.hindawi.com

\section{World Journal}

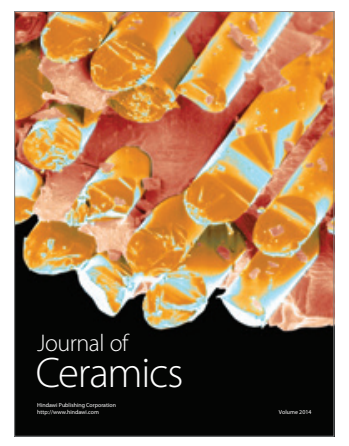

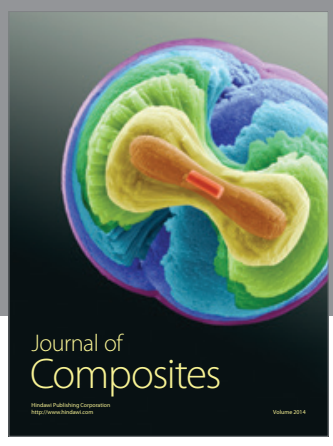
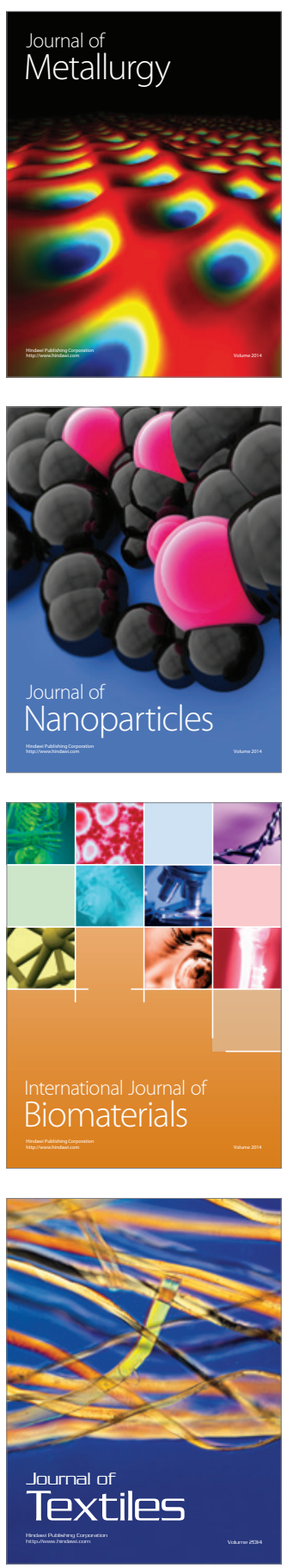